\title{
Coronary Endarterectomy for Diffuse Coronary Artery Disease in A Bangladeshi Population Cohort
}

\author{
MASOOM SIRAJ $^{1}$, MD HAMIDUR RAHMAN ${ }^{2}$, MD SHARIF HASSAN ${ }^{1}$ \\ ${ }^{1}$ Department of Cardiac Surgery, Ibrahim Cardiac Hospital and Research Institute, Dhaka, ${ }^{2}$ Department of Cardiac Anaesthesiology, \\ Ibrahim Cardiac Hospital and Research Institute, Dhaka
}

Address for correspondence: Dr. Masoom Siraj, Senior Consultant and Head of Cardiac Surgery, Ibrahim Cardiac Hospital and Research Institute, Shahbag, Dhaka - 1000

\begin{abstract}
:
Coronary artery bypass grafting (CABG) is a well established treatment modality for coronary artery disease (CAD). However with the trend towards aggressive per cutaneous interventions (PCI) by the cardiologists, more and more patients with poor quality, diffusely diseased coronary arteries are coming for CABG. Quite often these arteries require endarterectomy to ensure revascularisation. Initial experience world wide with coronary endarterectomy was bad enough for many surgeons not advocating it. However recent papers have shown greatly improved results.

This was a retrospective study of five hundred consecutive patients undergoing CABG between $19^{\text {th }}$ August 2006 and $1^{\text {st }}$ of July 2008 at Ibrahim Cardiac Hospital and Research Institute (ICHRI). Pre-operative and Intra-operative variables which could influence outcome were analysed. Of the total patients who had at least one endarterectomy done were labeled as Endarterectomy (EA) group, while patients without endarterectomy were labeled as Control group.

Post operative outcome showed results comparable to CABG without endarterectomy can be achieved. We have described our selection criteria and surgical technique. Our protocol did not bring about a statistically significant increase in bypass time, cross clamp time. It also did not change the number of grafts per patient.

Our experience shows coronary endarterectomy can be done in order to achieve full revascularisation with very safe and acceptable outcome.
\end{abstract}

Key words: $C A B G$ Coronary endarterectomy

\section{Introduction:}

The incidence of coronary artery disease is on an increasing trend. The profile of patients coming for Coronary artery bypass grafting (CABG) is also continuously changing to include older patients with associated co morbidities such as diabetes mellitus, hypertension, cigarette smoking, peripheral vascular disease, and others. ${ }^{1}$ With the increasing use of nonsurgical methods of coronary revascularization like Per Cutaneous Interventions (PCI) stenting or other catheterbased procedures, now patients with severe diffuse coronary disease are being referred for surgery, when the affected vessels are usually not graftable and complete revascularization using conventional CABG may not be feasible. It is well documented that incomplete myocardial revascularization procedure adversely affects the outcome of CABG. ${ }^{2-7}$ The option left to surgeons in this group of patients is to place a graft on the diffusely diseased vessel along with endarterectomy (EA) of the vessel. However, many surgeons are reluctant to perform endarterectomy because of the poorer clinical outcome $3,5,8-18,21$ as compared to conventional CABG. They are further dissuaded by the technical difficulty and uncertainty surrounding the procedure. Recently there has been renewed interest in the procedure after the publication of papers stating judicious use of endarterectomy can give appreciable results with good long-term outcome. ${ }^{19,20}$ Here we present our experience with Endarterectomy at Ibrahim Cardiac Hospital and Research Institute.

Materials and Methods:

In this analysis, retrospective data of 500 consecutive CABG cases between $19^{\text {th }}$ August 2006 to $1^{\text {st }}$ July 2008 were included. Key baseline variables were age, sex, LVEF, and number of coronary vessels involved. Risk factors studied included hypertension, diabetes mellitus, tobacco smoking, dyslipidaemia, preoperative MI, renal impairment, previous CVA, family history of IHD. Peroperative variables considered to have influence on the outcome of endarterectomy were CPB time, X-clamp time and number of grafts required. Outcome was evaluated with the help of postoperative arrhythmia, bleeding, perioperative MI and mortality. Of the total patients who had at least one endarterectomy done were labeled as Endarterectomy (EA) group, while patients without endarterectomy were labeled 
as control group. Patients having a concomitant procedure along with the CABG were excluded. All the patients were operated on by a single surgeon. The anaesthesia, perfusion, surgical and postoperative care team also remained the same for each case.

In all cases the following standard protocol was followed. Anaestheia was induced and maintained using Fentanyl, Etomidate, Thiopentone, Pancrunonium and propofol. Heart was approached via median sternotomy. Patients were attached to a Heart-Lung machine (Stockert III) using aortic and bicaval venous cannulation. Patients were cooled down to 28 degree celsius core temperature. Aorta was cross clamped and the heart was arrested with antegrade cold blood cardioplegia and topical cooling. Patients were rewarmed after removal of the cross clamp at the time of constructing the top ends. At the end of the procedure patients were disconnected from the Heart-Lung machine, closed and shifted to the cardiac surgical ICU. Majority of the patients were extubated within three hours.

The idea of elective endarterectomy was not practiced. Endarterectomy was done only when the vessel quality did not permit the construction of a meaningful graft during surgery. That is when the lumen failed to admit a $1 \mathrm{~mm}$ metal probe or the lumen was filled with clots, debris that could not be cleared. A very conservative approach was adopted for Left Anterior Descending (LAD) endarterectomy. The viability of the supplying territory was also considered at the time of making a decision. In majority of the cases endarterectomy was achieved using a limited arteriotomy and the "traction" technique. 15,22,23 When the endarterectomy was judged as inadequate additional arteriotomy was made or it was converted to the “open method” of endarterectomy. ${ }^{15,24}$ Left Internal Mammary Artery (LIMA) was anastomosed to the Left Anterior Descending (LAD) artery whenever possible. We do not utilize the LIMA for sequential anastomosis. A reversed vein graft was substituted for the LAD whenever needed. In all case the conduit was directly anastomosed to the endarterectomy site.

\section{Results:}

Of the demographic characteristics age was fairly comparable between the endarterectomy and control groups ( $56.2 \pm 8.0$ vs. $55.7 \pm 8.8, \mathrm{p}=0.703)$. However, female sex demonstrated their significant presence in the endarterectomy group (36.4\%) than that in the control group $(14.3 \%)(p<0.001)$ as shown in Table I. Over $63 \%$ of the endarterectomy group had LVEF $>50 \%$ (preserved) as opposed to $45.8 \%$ of the control group (Fig.-1). All the patients who underwent endarterectomy had experience of prior CVA. Presence of hypertension and history of previous renal failure were also significantly higher in the endarterectomised patients (Table-II) compared to those who underwent CABG without endarterectomy $(\mathrm{p}=0.020$ and $\mathrm{p}=0.024$ respectively).

Family history of ischemic heart diseases was much less in the endarterectomy group than that in the latter group ( $p<0.001)$. Perioperative factors (Table-III) that might influence the outcome were all identically distributed in both the groups. Immediate outcome (Table-IV) of endarterectomy did not differ with that of control group except in terms of arrhythmia. Which was considerably higher in the EA group (9\%) than that in the control group (2.6\%) and this was significant $(\mathrm{p}<0.001)$.

Table-I

Distribution of demographic characteristics between case and control groups

\begin{tabular}{lccc}
\hline \multirow{2}{*}{$\begin{array}{l}\text { Demographic } \\
\text { characteristics }\end{array}$} & \multicolumn{2}{c}{ Group } & p- \\
\cline { 2 - 3 } & Endarterectomy & Control & value \\
\hline Age & $(\mathrm{n}=55)$ & $(\mathrm{n}=496)$ & \\
$\leq 50$ & $15(27.3)$ & $146(29.4)$ & \\
$>50$ & $40(72.7)$ & $350(70.6)$ & \\
Mean \pm SD years ${ }^{\#}$ & $56.2 \pm 8.0$ & $55.7 \pm 8.8$ & 0.703 \\
Sex & & & \\
Male & $35(63.6)$ & $425(85.7)<0.001$ \\
Female & $20(36.4)$ & $71(14.3)$ & \\
\hline
\end{tabular}

\# Data analysed using Student's t-Test and presented as mean \pm SD.

I Data analysed with the help of Chi-square Test $\left(c^{2}\right)$ and presented as n (\%).

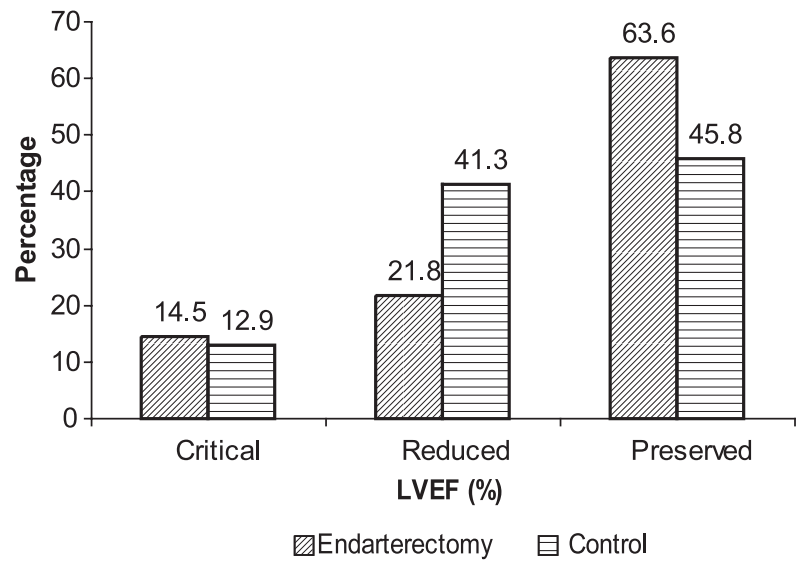

Fig.-1: Comparison of LVEF between endarterectomy and control groups 
Table-II

Distribution of risk factors between the groups

\begin{tabular}{lccc}
\hline Risk factors & \multicolumn{2}{c}{ Group } & $\begin{array}{c}\mathrm{p}- \\
\text { value }\end{array}$ \\
\cline { 2 - 3 } & $\begin{array}{c}\text { Endarterectomy Control } \\
(\mathrm{n}=55)\end{array}$ & $\begin{array}{c}\mathrm{n}=496) \\
\text { val }\end{array}$ \\
\hline PriorCVA & $55(100.0)$ & $1(0.2)$ & $<0.001$ \\
Perioperative MI & $0(0.0)$ & $0(0.0)$ & - \\
Previous renal impairment & $12(21.8)$ & $56(11.3)$ & 0.024 \\
Diabetes mellitus & $36(65.5)$ & $292(58.9)$ & 0.345 \\
Hypertension & $49(89.1)$ & $372(75.0)$ & 0.020 \\
Smoking & $26(47.3)$ & $241(48.8)$ & 0.831 \\
Dyslipidaemia & $36(65.5)$ & $292(58.9)$ & 0.345 \\
Family history IHD & $3(5.5)$ & $138(27.8)$ & $<0.001$ \\
Hypothyroidism & $1(1.8)$ & $2(0.4)$ & 0.176 \\
\hline
\end{tabular}

Table-III

Perioperative factors influencing outcome of endarterectomy

\begin{tabular}{lccc}
\hline Perioperative factors & \multicolumn{2}{c}{ Group } & \begin{tabular}{c}
$\mathrm{p}$ - \\
\cline { 2 - 3 }
\end{tabular} \\
\cline { 2 - 3 } & $\begin{array}{c}\text { Endarterectomy Control } \\
\text { value }\end{array}$ \\
& $(\mathrm{n}=55)$ & $(\mathrm{n}=496)$ & \\
\hline CPB time(minutes) & $110.9 \pm 44.8$ & $97.1 \pm 96.9$ & 0.299 \\
X-clamp time(minutes) & $66.9 \pm 21.4$ & $60.3 \pm 17.4$ & 0.650 \\
Number of grafts required & $3 \pm 1$ & $3 \pm 1$ & 0.861 \\
\hline
\end{tabular}

\# Data analysed using Student’s t-Test and presented as mean \pm SD.

Table-IV

Immediate outcome of endarterectomy

\begin{tabular}{|c|c|c|c|}
\hline \multirow{2}{*}{ Outcome variables } & \multicolumn{2}{|c|}{ Group } & \multirow{2}{*}{$\begin{array}{c}\text { p- } \\
\text { value } \\
\end{array}$} \\
\hline & $\begin{array}{l}\text { Endarterectomy } \\
\qquad(\mathrm{n}=55)\end{array}$ & $\begin{array}{l}\text { Control } \\
(n=496)\end{array}$ & \\
\hline Postoperative arrhythmia ${ }^{\#}$ & $5(9.0)$ & $13(2.6)$ & $<0.001$ \\
\hline Postoperative bleeding\# & $1(1.8)$ & 7(1.4) & 0.647 \\
\hline Perioperative MI & 2(3.6) & $10(2.0)$ & 0.161 \\
\hline Perioperative mortality & $1(1.8)$ & $6(1.2)$ & 0.529 \\
\hline
\end{tabular}

\# Data analysed using Chi-square $\left(\chi^{2}\right)$ Test and were presented as mean

\section{Discussion}

The technique of coronary artery endarterectomy was developed by Bailey and colleagues ${ }^{6}$ in the early days of CABG. ${ }^{3}$ However the technique did not get popular because of the technical difficulties involved in the process of endarterectomy and the higher incidence of adverse clinical events..$^{3,5,8-18}$ But there has been a change in attitude mainly brought about the referral of patients with severe diffuse coronary artery disease. These patients are not deemed to be suitable for PCI and only options left for them are endarterectomy.

The present study revealed that females are more likely to have diffuse coronary artery disease than their male counterpart requiring an endarterectomy. Previous CVA, hypertension and renal impairment were significantly common in the endarterectomy group than those in the control group suggesting these factors might be associated with diffuse coronary artery disease. Diabetes, dyslipidaemia and smoking were not found to carry extra risk for diffuse coronary artery disease in our Experience. Although other investigators have found them as risk factors. In our series the immediate outcome of endarterectomy is encouraging. Postoperative bleeding, MI and mortality were all negligible and did not differ from the control group. Only arrhythmia was significantly higher in the endarterectomy patients which were managed medically. All this justifies performing endarterectomy is better than doing an incomplete revascularisation.

Despite increased risks involved a better outcome is being increasingly reported by judicious selection of target vessel for performing endarterectomy. There is no uniformity in the frequency of coronary endarterectomy. ${ }^{26}$ Some centers do not entertain the procedure at all. Others who have adopted the procedure report an incidence between 3.7\% and $42 \%{ }^{8,26}$ The reported incidence of mortality after EA ranges between $3.2 \%$ and $10 \%$ and that of myocardial infarction (MI) is between $4 \%$ and $15 \%$. $3,5,8-18$

Newer studies report outcomes very similar to CABG without EA. This has been possible by a combination of careful patient selection, improved anaesthesia, myocardial protection, sophisticated surgical technique and better postoperative management. There are studies showing increased operative risk for LAD endarterectomy ${ }^{7,16}$ as compared to the Right Coronary Artery (RCA). A number of studies $^{16,18,25}$ have shown improved outcome when the LIMA is used to reconstruct the enadarterectomised vessel.

Our study shows a mortality of $1.8 \%$ for endarterectomy during coronary artery bypass. This is well within the worldwide standard and justifies against not doing the procedure. Our selection criteria further shows the procedure can be done with a bypass time and crossclamp time statistically not significantly different from a straightforward coronary bypass. In our study female sex and a history of previous CVA was statistically significant 
enough to warn off the surgeon of bad quality vessels with a potential need for endarterectomy during surgery.

The study concludes that patients with diffuse coronary artery disease are high risk candidates for revascularization. However with careful selection of patients, well-judged and a well executed surgical technique, followed by close postoperative care very good results can be obtained.

\section{References:}

1. The Society of Thoracic Surgeons Database. The eighth year. Minnetonka, MN: Summit Medical, December 1998.

2. Schaff HV, Gersh BJ, Pluth JR, et al. Survival and functional status after coronary artery bypass grafting: results of 10-12 years after surgery in 500 patients. Circulation 1983; 68 (Suppl II): 200-04.

3. Loop FD. Resurgence of coronary artery endarterectomy. J Am Coll Cardiol 1988; 11: 712-13.

4. Parsonnet V, Gilbert L, Gielchinsky I, Bhaktan EK, Atherley T. Coronary endarterectomy: an analysis of its role in aortoCoronary endarterectomy (CE) is an important tool for the cardiac surgeon, because the number of patients with severe coronary artery disease continues to in-crease. To identify risk factors for adverse outcome coronary bypass. Cardiovasc Dis Bull Texas Heart Inst 1976; 3: 51-60. INVITED COMMENTARY

5. Miller DC, Stinson EB, Dyer PE, et al. Long-term clinical assessment of efficacy of adjunctive coronary endarterectomy. J Thorac Cardiovasc Surg 1981; 81: 21-29.

6. Bailey CP, May A, Lemmon WM. Survival after coronary endarterectomy in man. JAMA 1957; 164: 641-46.

7. Longmire WP, Cannon JA, Kattus AA. Directvision coronary endarterectomy for angina pectoris. N EngI J Med 1958; 259: 993-99.

8. Qureshi SA, Halim MA, Pillai R, Smith P, Yacoub MH. Endarterectomy of the left coronary system. Analysis of 10 year experience. J Thorac Cardiovasc Surg 1985; 89: 852-59.

9. Livesay JL, Cooley DA, Hallman GL, et al. Early and late results of coronary endarterectomy. Analysis of 3369 patients. J Thorac Cardiovasc Surg 1986; 92: 649-57.

10. Bernowitz JB, Kayser KL, Johnson WD. Results of coronary artery endarterectomy and reconstruction. J Thorac Cardiovasc Surg 1988; 95: 1-10.

11. Ladowski JS, Schatzlein MH, Underhill DJ, Peterson AC. Endarterectomy, vein patch, and mammary bypass of the anterior descending artery. Ann Thorac Surg 1991; 52: 1187-89.
12. Goldstein J, Cooper E, Saltups A, Boxall J. Angiographic assessment of graft patency after coronary endarterectomy. J Thorac Cardiovasc Surg 1991; 102: 539-45.

13. Christakis GT, Rao V, Fremes SE, Chen E, Naylor D, Goldman BS. Does coronary endarterectomy adversely affect the result of bypass surgery? J Cardiac Surg 1993; 8: 72-78.

14. Johnston RH, Garcia-Rinaldi R, Wall MJ Jr. Coronary artery endarterectomy: a method of myocardial preservation. Texas Med 1993; 89: 56-59.

15. Goldman BS, Christakis GT. Endarterectomy of the left anterior descending coronary artery. J Cardiac Surg 1994; 9: 89-96.

16. Djalilian AR, Shumway SJ. Adjunctive coronary endarterectomy: improved safety in modern cardiac surgery. Ann Thorac Surg 1995; 60: 1749-54.

17. Tasdemir O, Kiziltepe U, Karagoz HY, Yamak B, Korkmaz S,Bayazit K. Long-term results of reconstructions of the left anterior descending coronary artery in diffuse atherosclerotic lesions. J Thorac Cardiovasc Surg 1996; 112: 745-54.

18. Gill IS, Beanlands DS, Boyd WD, Finlay S, Keon WJ. Left anterior descending endarterectomy and internal thoracic artery bypass for diffuse coronary disease. Ann Thorac Surg 1998; 65: 659-62.

19. Sundt TM III, Camillo CJ, Mendeloff EN, Barner HB, Gay WA Jr. Reappraisal of coronary endarterectomy for the treatment of diffuse coronary artery disease. Ann Thorac Surg 1999; 68: 1272-77.

20. Marinelli G, Chiappini B, Di Eusanio M, et al. Bypass grafting with coronary endarterectomy: immediate and long-term results. J Thorac Cardiovasc Surg 2002; 124: 553-60.

21. Minale C, Nikol S, Zander M, Uebis R, Effert S, Messmer BJ. Controversial aspect of coronary endarterectomy, Ann Thoracic Surg 1989; 48: 235-41.

22. Mills NE. Coronary endarterectomy. Adv Cardiac Surg 1998; 10: $197-227$.

23. Parsonnet V, Gilbert L, Gielchinsky I, Bhaktan EK. Endarterectomy of the left anterior descending and main stem coronary arteries: a technique for reconstruction of inoper-able arteries. Surgery 1976; 80: 662-72.

24. Sellke FW, Laham RJ, Edelman ER, Pearlman JD, Simons M. Therapeutic angiogenesis with basic fibroblast growth fac-tor. Ann Thorac Surg 1998; 65: 1540-44.

25. Beretta L, Lemma M, Vanelli P. Coronary “open” endarterectomy and reconstruction. Eur J Cardiothorac Surg 1992; 6: 382-87.

26. Atik FA, Dallan LA, de Oliveira SA. Myocardial revascularization with coronary endarterectomy. Stratification of risk factors for early mortality. Arq Bras Cardiol 2000; 75: 269-80. 